\title{
Systemic Approaches Identify a Garlic-Derived Chemical, Z-ajoene, as a Glioblastoma Multiforme Cancer Stem Cell-Specific Targeting Agent
}

\author{
Yuchae Jung ${ }^{1,2}$, Heejoo Park ${ }^{1,2}$, Hui-Yuan Zhao ${ }^{1}$, Raok Jeon ${ }^{1}$, Jae-Ha Ryu ${ }^{1}$, and Woo-Young Kim ${ }^{1, *}$
}

Glioblastoma multiforme (GBM) is one of the most common brain malignancies and has a very poor prognosis. Recent evidence suggests that the presence of cancer stem cells (CSC) in GBM and the rare CSC subpopulation that is resistant to chemotherapy may be responsible for the treatment failure and unfavorable prognosis of GBM. A garlic-derived compound, Z-ajoene, has shown a range of biological activities, including anti-proliferative effects on several cancers. Here, we demonstrated for the first time that Z-ajoene specifically inhibits the growth of the GBM CSC population. CSC sphere-forming inhibition was achieved at a concentration that did not exhibit a cytotoxic effect in regular cell culture conditions. The specificity of this inhibitory effect on the CSC population was confirmed by detecting CSC cell surface marker CD133 expression and biochemical marker ALDH activity. In addition, stem cell-related mRNA profiling and real-time PCR revealed the differential expression of CSC-specific genes, including Notch, Wnt, and Hedgehog, upon treatment with Z-ajoene. A proteomic approach, i.e., reverse-phase protein array (RPPA) and Western blot analysis, showed decreased SMAD4, p-AKT, 14.3.3 and FOXO3A expression. The protein interaction map (http://string-db.org/) of the identified molecules suggested that the AKT, ERK/p38 and TGF $\beta$ signaling pathways are key mediators of Z-ajoene's action, which affects the transcriptional network that includes FOXO3A. These biological and bioinformatic analyses collectively demonstrate that Z-ajoene is a potential candidate for the treatment of GBM by specifically targeting GBM CSCs. We also show how this systemic approach strengthens the identification of new therapeutic agents that target CSCs.

\footnotetext{
${ }^{1}$ Research Center for Cell Fate Control (RCCFC) and College of Pharmacy, Sookmyung Women's University, Seoul 140-742, Korea, ${ }^{2}$ These authors contributed equally to this work.

*Correspondance: wykim@sookmyung.ac.kr
}

Received 9 June, 2014; revised 3 July, 2014; accepted 4 July, 2014; published online 31 July, 2014

Keywords: ajoene, CSC, garlic, GBM

\section{INTRODUCTION}

Glioblastoma multiforme (GBM) is one of the most common and highly lethal brain malignancies (Bielen et al., 2011). GBM patients have a poor prognosis and a median survival of approximately one year after diagnosis (Bielen et al., 2011). The poor clinical outcome of the disease is unlikely to be overcome by conventional treatment, emphasizing the need to develop novel therapeutic strategies (Eyler et al., 2011).

Increasing evidence suggests that GBM contains a highly tumorigenic subpopulation of cells that is responsible for tumor initiation and progression (Hyun et al., 2011). Because these cells shows stem cell-like properties, such as self-renewal and differentiation into other cell types, these cells are referred to as cancer stem cells (CSC) or tumor initiating cells (Soeda et al., 2009). Largely due to the dormant nature of stem cells, CSCs are resistant to chemo- and radiotherapy (Dean et al., 2005; Gangemi et al., 2009). Although conventional chemoregimens combined with aggressive radiation may kill the majority of cancer cells in a tumor mass, the treatment-resistant CSC population continues to survive and self-renew, ultimately leading to treatment failure and recurrence of the cancer (Hyun et al., 2011). Therefore, surgical resection with combined therapy for CSCs may be a potential solution to eradicate this malignant cancer (Lu et al., 2011).

Z-ajoene was first isolated from processed garlic in an E/Zmixture (Block et al., 1984; Kaschula et al., 2012). It has been reported that Z-ajoene functions as an antioxidant agent and may be a potent antithrombotic agent (Kaschula et al., 2012). It has also been reported that Z-ajoene has anti-proliferative effects on several cancer cells at high concentration ( $\mathrm{Li}$ et al., 2002; Taylor et al., 2006). However, the effects of Z-ajoene on the CSC population have not yet been reported. In this study, we examined the potential of Z-ajoene to specifically target GBM CSCs and found that it suppressed anti-GBM CSC sphere growth. Tests for known CSC markers revealed that Zajoene treatment might specifically target CSC activity. We also investigated the mechanism of this anti-CSC effect using a proteomics approach (reverse-phase protein array, RPPA). The molecules identified by mRNA profiling, real-time PCR, RPPA and western blot analysis were analyzed using bioinformatics approaches to unveil the systemic action of Z-ajoene in CSCs of GBM. 


\section{MATERIALS AND METHODS}

\section{Reagents and cells}

Z-ajoene was prepared as described previously (Lee da et al., 2012). The human GBM cell lines U87 and U251 were obtained from the American Type Culture Collection (ATCC, USA) and Dr. Mark Johnson (Brigham and Women's Hospital), respectively. OCT3/4-promoter-EGFP-transfected U87 cells were gifted from Dr. Hyunggee Kim (Korea University).

\section{CSC sphere culture}

PolyHEMA-(poly-2-hydroxyethyl methacrylate) (Sigma Aldrich, USA) was dissolved at a concentration of $5 \mathrm{mg} / \mathrm{ml}$ in $95 \%$ ethanol and overlaid on the surfaces of culture plates followed by complete drying. GBM cells were then resuspended in DMEM/ F12 medium (Invitrogen, USA) containing $20 \mathrm{ng} / \mathrm{ml}$ epidermal growth factor (EGF), basic fibroblast growth factor (bFGF) and B27 neural supplement (Invitrogen), in addition to 100 units $/ \mathrm{ml}$ penicillin and $100 \mu \mathrm{g} / \mathrm{ml}$ streptomycin.

\section{CSC sphere formation inhibition assay}

U87 cells (1000 cells/well) were seeded and treated with different concentrations of Z-ajoene in a 384-well black plate (Corning, USA). On the 3rd day, the compound was included with fresh media, which was added to each well. After 2 more days, Hoechst 33342 dye was added to each well, and an image reader Cytation3 (Biotek, USA). was used for quantitative sphere counting ( $\geq 100 \mu \mathrm{m}$ ). Each experiment was repeated in 14 wells, and each data point represents the mean and standard error, as shown in the "Results".

\section{Real-time RT-PCR analysis and stem cell RNA profiling} Total RNA was extracted using the TRI reagent (MRC, Inc., USA). Real-time PCR was performed using the primer sequences listed in Supplementary Table 1. The expression level of each mRNA was normalized to the expression level of GAPDH or L32.

For stem cell RNA profiling, RNA was reverse transcribed into cDNA using the $\mathrm{RT}^{2}$ First-strand cDNA synthesis kit (Qiagen, USA) and transferred to each PCR array plate (Qiagen). Analyses were performed in triplicate using the Step-One Plus Real-Time PCR System (Applied Biosystems, USA). The expression level of each mRNA was normalized to the average expression of 4 housekeeping genes: GAPDH, B2M, $\beta$-actin, and HPRT1. The normalized data were then analyzed using the web-based software provided by QIAGEN (http:// www.sabiosciences.com/pcrarraydataanalysis.php). P-values were calculated using T-test.

\section{CSC markers FACS assay}

U87 $\left(5 \times 10^{5}\right.$ cells $\left./ \mathrm{ml}\right)$ was seeded and treated with DMSO or $2.5 \mu \mathrm{M}$ Z-ajoene using sphere forming conditioned in $100 \mathrm{~mm}$ dishes. After 5 days, the dissociated cells were incubated with CD133-PE antibody (Miltenyi Biotec, USA) supplemented with FcR Blocking Reagent, human (Miltenyi Biotec). Cells were analyzed by flow cytometry (FACS Calibur ${ }^{\mathrm{TM}}$, BD Bioscience). For ALDF assay, the dissociated CSC was incubated with ALDEFLUOR $^{\mathrm{TM}}$ reagent (StemCell Technologies, USA). ALDH positive cells were analyzed by flow cytometry (FACSCalibur ${ }^{\mathrm{TM}}$ ).

RPPA and Western blot analysis

U87 cells were cultured in sphere forming condition and treated with Z-ajoene for $12 \mathrm{~h}$ and total lysates were analyzed by MD Anderson core facility for RPPA. The clustering of samples and generation of heat map were performed by using the Cluster and TreeView programs. For Western blot, lysates were prepared from the cells in monolayer or spherical culture condition. Antibodies to p-Erk, p-p38, p-pS6, p-AKT(Ser-473), p-AKT(Thr308) and p-GSK3 were obtained from Cell Signaling Technology (USA). Antibodies to SMAD4, Erk1 and GAPDH were from Santa Cruz (USA).

\section{RESULTS}

\section{Inhibitory effect of glioblastoma CSC sphere growth by}

Z-ajoene

To examine the anti-CSC activity of Z-ajoene, we first optimized CSC-enriched culture conditions after coating cell culture plates with poly-HEMA (Fukazawa et al., 1995). U87 cells formed typical spheres, and cells transfected with the OCT3/4p-EGFP plasmid (Gerrard et al., 2005) expressed EGFP mainly in the surfaces cell layers, suggesting that the stem-like cells were enriched in the spheres. The mRNA expression of CD133, an extensively used GBM cancer stem cell surface marker (Singh et al., 2004), increased in the spherical culture compared to cells cultured in the serum-containing medium (Fig. 1B). The cells expressing the CD133 marker on the cell surface were also enriched in our CSC culture condition (Fig. 1C). We then employed this culture condition using hydrophobic coating and conditioned medium for the screening of the anti-CSC activity of the selected Z-ajoene.

Previously, it was reported that Z-ajoene inhibited leukemia and melanoma cell growth at relatively high concentrations, 10$40 \mu \mathrm{M}$ (Dirsch et al., 1998; Taylor et al., 2006). We examined whether Z-ajoene could disrupt the sphere-forming activity of GBM CSCs (Fig. 1D). Z-ajoene treatments of the U87 GBM cell line at $2.5 \mu \mathrm{M}$ and $5 \mu \mathrm{M}$ did not significantly inhibit cell growth in the regular monolayer culture condition (closed circle in Fig. 1D). However, the treatment substantially inhibited the sphereforming activity of the cells in a dose-dependent manner at the same doses (opened circle in Fig. 1D). Z-ajoene also showed a similar inhibitory growth effect on CSCs of another GBM cell line, U251, suggesting that the observed anti-CSC sphere effect of Z-ajoene was not cell line-specific (Fig. 1E).

\section{Selective suppression of GBM CSC marker-expressing cells by Z-ajoene}

To further validate the specific inhibitory effect of Z-ajoene on the sphere-forming activity of GBM CSCs, we performed flow cytometry analyses using the CD133 cell surface antigen, which is believed to be the most robust surface marker for GBM CSCs, after 5 days of Z-ajoene treatment. The CD133 ${ }^{+}$subpopulation in the CSC culture condition was significantly decreased in Z-ajoene-treated cells (from $78.0 \pm 0.3 \%$ to $48.2 \pm$ $0.37 \%$, Fig. $2 A$ ).

We also employed the ALDEFLUOR ${ }^{\mathrm{TM}}$ assay, which measures the activity of the CSC-predominant enzyme, ALDH (aldehyde dehydrogenase), which is one of the most widely used CSC markers (Ginestier et al., 2007). DEAB (diethylaminobenzaldehyde), an inhibitor of ALDH, was used as a negative control. Cells treated with DMSO (control) or $2.5 \mu \mathrm{M}$ Z-ajoene were analyzed using flow cytometry and ALDEFLUOR staining (Fig. 2B). After treatment with $2.5 \mu \mathrm{M}$ Z-ajoene, the ALDH-positive cells decreased to $6.2 \%$, compared with $14.2 \%$ for the DMSOtreated control group. These results support our finding that Zajoene specifically decreases the CSC population in GBM. 
A

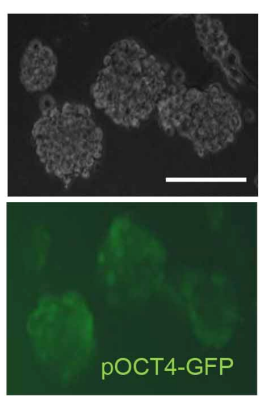

B

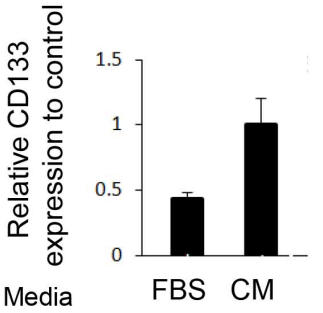

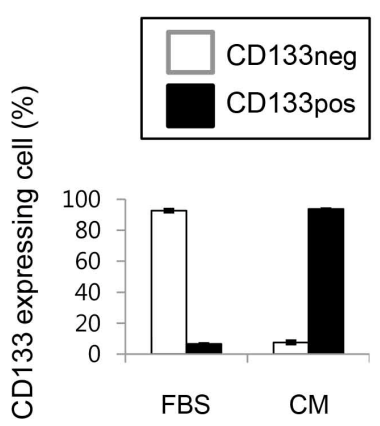
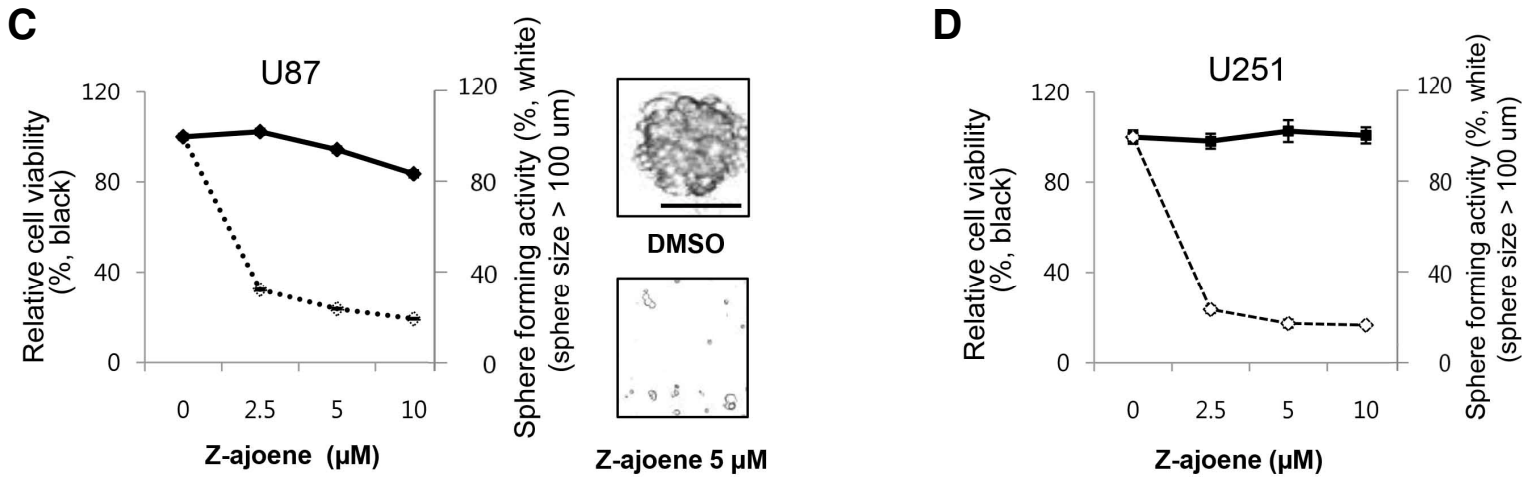

Fig. 1. Z-ajoene suppresses CSC sphere formation (A) EGFP-OCT3/4 expressing cells in U87-derived neurosphere, (B) CD133 mRNA expression level by semi-quantitative RT-PCR (left) and CD133 positive population by FACS analysis (right) showed that the CSC population increased in the spherical culture condition. (C, D) The effect of Z-ajoene on GBM cells, U87 (C) and U251 (D) in attached or spherical culture condition. DMSO was used as control. Spheres with $100 \mu \mathrm{m}$ of diameter were only counted. Error bars represent standard errors.
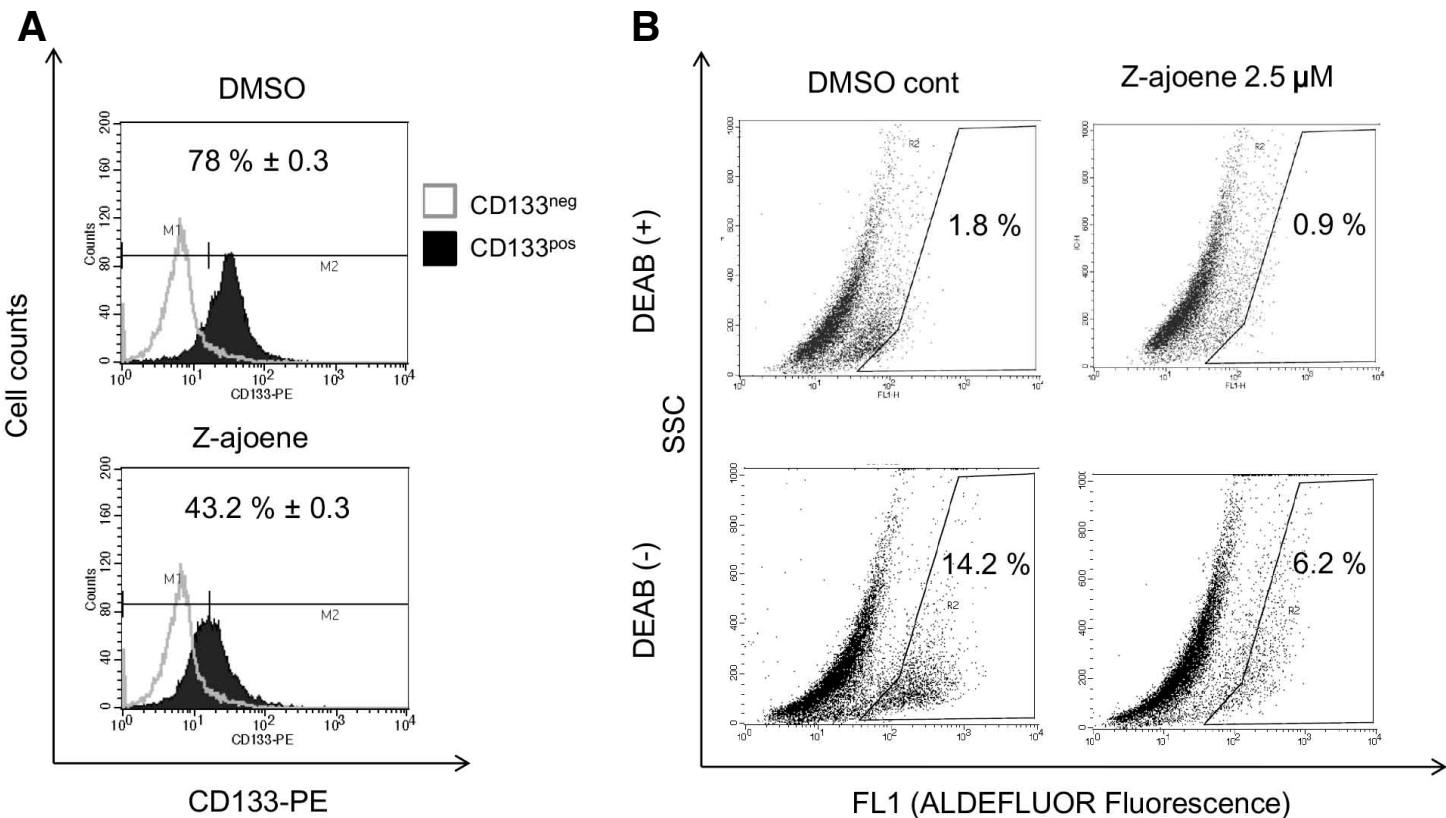

Fig. 2. Z-ajoene treatment suppresses GBM U87 CSC. (A) Decrea-se of CD133 positive cells by Z-ajoene. The spheres were dissociated and labeled with CD133-PE-Ab followed by FACS analysis. (B) Decrease of ALDH positive cells by Z-ajoene. The sphere cells were dissociated to single cells and then incubated with ALDE-FLUOR substrate without or with DEAB, a specific inhibitor of ALDH. 

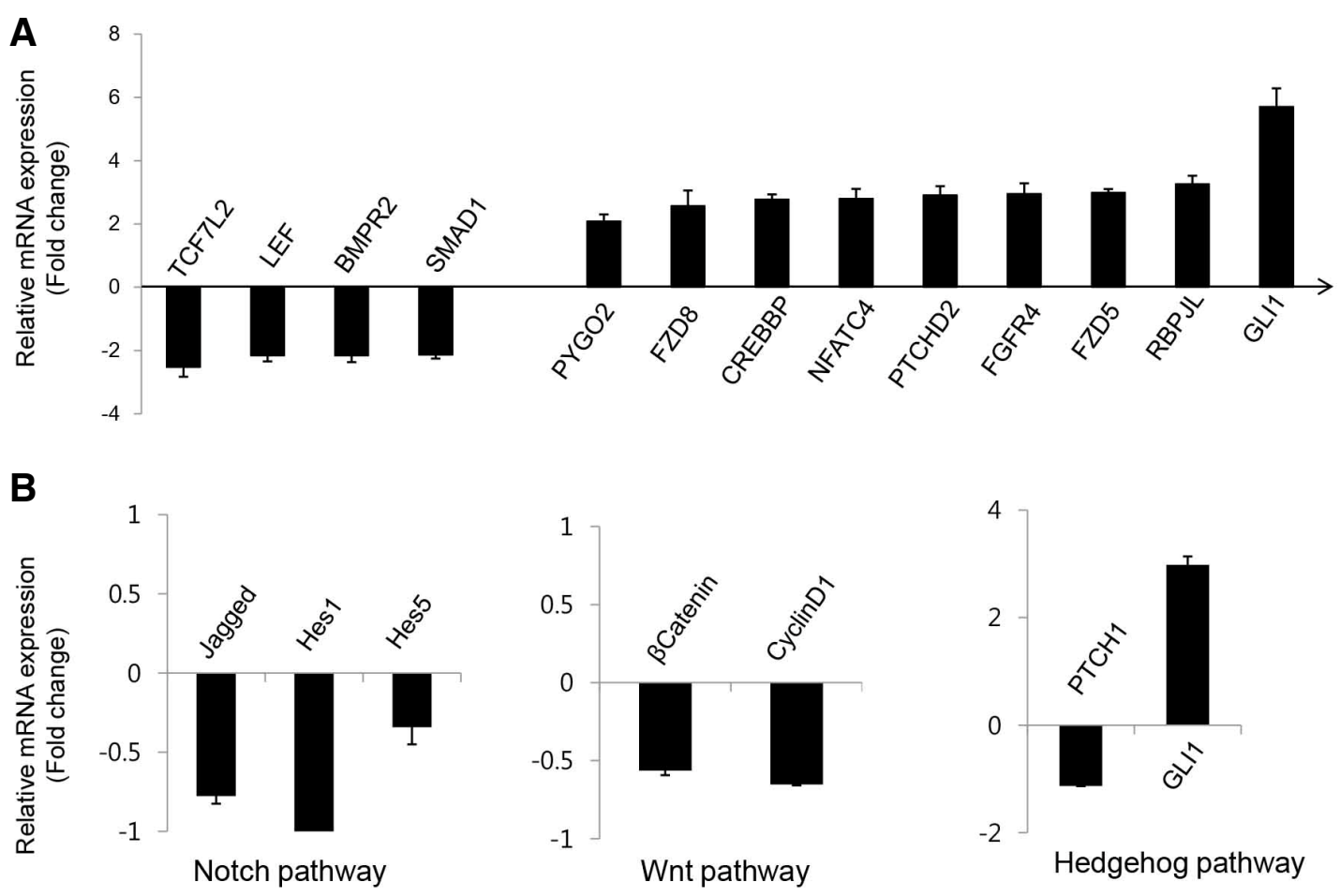

Fig. 3. Gene expression changes in CSC sphere cells by Z-ajoene in $12 \mathrm{~h}$. (A) Stem cell focused quantitative-PCR array revealed 13 genes, the expression is bigger than 2 fold or smaller then $1 / 2$ of control with $P<0.05$. (B) Real-time PCR of Notch, Wnt and Hedgehog downstream genes.

\section{Modulation of stem cell-related gene expression by Z-ajoene}

We further investigated whether the expression levels of signaling molecules known to control stem cell destiny were modulated by Z-ajoene after $12 \mathrm{~h}$ of Z-ajoene treatment, which was far earlier then when the decreases in cell number occurred (Fig. 3). We employed a genomics approach, i.e., a quantitative RT-PCR array for 84 known stem cell genes (http://www.sabiosciences.com/). The most significantly changed molecules are shown in Fig. 3A. As expected from the subsequent loss of the CSC population, the expression of several key molecules that regulate stem cell survival and maintenance were significantly modulated at this early time of treatment (Malhotra et al., 2011). In triplicate experiments, Z-ajoene significantly increased the mRNA expression of GLI1, RBPJL, FZD5, FGFR4, PTCHD2, NFATC4, CREBBP, FZD8, PYGO2 and NOTCH3. Unexpectedly, TCF7L2/LEF1 in the WNT pathway and BMPR2 and SMAD1 in the TGF $\beta$ signaling pathway were decreased by Zajoene. We further confirmed that the downstream targets of the Notch, WNT and Hedgehog signaling pathways were downregulated based on real-time PCR analyses (Fig. 3B). These results strongly suggested that the orchestrated signaling network in CSCs was modulated by Z-ajoene after $12 \mathrm{~h}$, and the following loss of the CSC population was most likely associated with the alterations of the signaling network caused by Z-ajoene.

Potential mechanisms mediating the anti-CSC activity of Z-ajoene, identified by systemic approaches We pursued a proteomic approach, RPPA, to identify the intracellular signaling that is modulated by Z-ajoene in CSC cells (clustered heat map for entire proteins is shown in Supplemen- tary Fig. 2). Of the 171 different validated antigens related to cancer (http://www.mdanderson.org), 8 molecules showed significant and clear differential expression $(P<0.05)$ upon treatment with Z-ajoene (Fig. 4A). These molecules included TGF $\beta$ signaling-related molecules (SMAD4, ACVRL), an AKT downstream effecter (FOXO3A) and a molecule that is known to be associated with CSC signaling (c-Kit) (Mani et al., 2008; Sunayama et al., 2011; Zhang et al., 2008). In addition, p-myosin, TIGAR, SF2, and 14.3.3, which have potential roles in CSC maintenance or differentiation, showed differential expression upon Z-ajoene treatment. We further performed western blot analysis for several molecules whose functions were related to the functions of the identified molecules (Fig. 4B). Consistent with the RPPA results, the level of SMAD4 protein was decreased, and the decreased FOXO3A expression demonstrated by RPPA was correlated with the downregulation of AKT signaling ( $\mathrm{p}-\mathrm{AKT}$-Ser473, $\mathrm{p}-\mathrm{AKT}-\mathrm{Thr} 308$, and $\mathrm{p}-\mathrm{pS6}$ ) by Zajoene as determined by western blot analysis. Because MAPK signaling was also found to have an important role in CSC maintenance and proliferation (Matsuda et al., 2012), we examined the expression of $p$-ERK and p-p38. Surprisingly, those signaling molecules were activated by Z-ajoene in CSCs but not in the regular monolayer culture condition. Interestingly, many of these changes were specifically shown in the CSC sphere culture condition but not in the monolayer culture condition, suggesting that the CSC population contains specific targeting machinery that is distinguishable from the regular cancer cell population. 


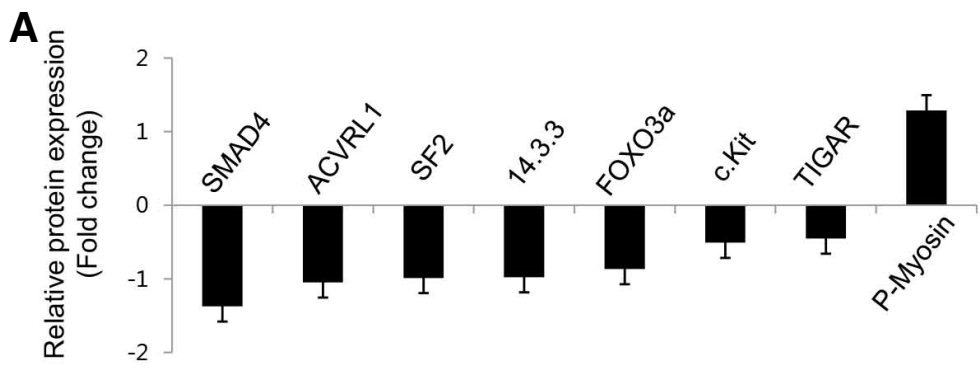

B

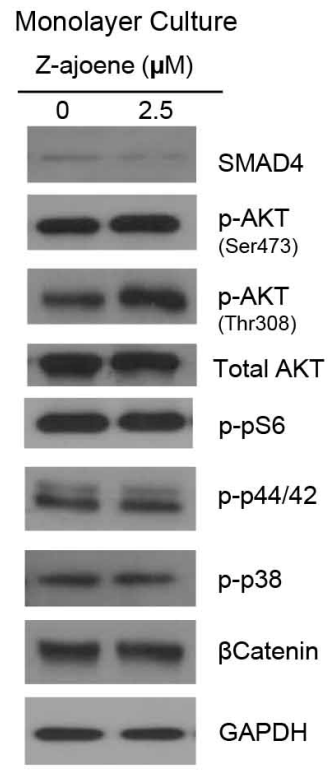

Fig. 4. Proteins expression changes in CSC sphere cells by Z-ajoene in $12 \mathrm{~h}$. (A) RPPA arrays results showing proteins express more than $2^{0.4}$ fold less than $2^{-0.4}$ fold of control with $P$ $<0.05$. (B) Western blotting of signaling molecules related to RPPA results.

\section{DISCUSSION}

Anticancer treatments that can specifically target CSCs and are less toxic to normal cells can be more effective against cancer than conventional, cytotoxic agent-based chemotherapies (Hyun et al., 2011; Yoon et al., 2012). In this study, we found that Zajoene inhibited the sphere-forming activity of the CSC population of GBM cells, which express CD133 and ALDH. Of note, this inhibitory effect on GBM CSCs was identified at a dose that did not significantly inhibit cell growth in the regular culture conditions of the GBM cell lines in vitro. These findings suggest that Z-ajoene can be used as a selective chemo-agent to specifically target CSC activities in GBM without damaging other cells, but this possibility requires further validation using in vivo tests.

In this study, the anti-CSC effect of Z-ajoene was mainly evaluated after 5 days of treatment, which was when the maximal responses of the GBM cell lines to Z-ajoene were observed (Figs. 1 and 2). Interestingly, the changes in the mRNA expression of known stem cell genes, especially involved in $\mathrm{NOTCH}$, WNT and Hedgehog signaling, were observed much earlier (i.e., $12 \mathrm{~h}$ after Z-ajoene treatment), suggesting that the antiCSC effects of Z-ajoene might be mediated by early transcriptional changes in the key stemness genes. Thus, it is expected that Z-ajoene treatment changes the transcription of key stemness genes, subsequently inducing the activation or deregulation of a number of cellular signaling pathways and their asso- ciated phenotypic changes. However, the modulation of the transcription of many genes by Z-ajoene in $12 \mathrm{~h}$ also suggests the deregulation of other molecular signaling pathways upstream of those transcriptional changes. The eight most-altered proteins of the related downstream effectors, as observed by RPPA and western blot analysis, revealed that TGF $\beta$ and AKT signaling are the main candidate signaling pathways targeted by Z-ajoene. Using the RPPA assay, we observed that the level of FOXO3A protein was also decreased following Z-ajoene treatment, which could be explained by the well-known signaling hierarchy of FOXO3A through AKT signaling, i.e., the failed sequestration of $\mathrm{FOXO} 3 \mathrm{~A}$ in the cytoplasm due to the dephosphorylation of AKT may be responsible for the decrease in FOXO3A in the RPPA results (Calnan and Brunet, 2008). The sharply decreased AKT activity, revealed by the reduced level of phosphorylated pS6 even before the phosphorylated form of AKT was marginally decreased (Fig. 4), suggested the possibility that Z-ajoene might directly target AKT activity or its downstream molecules, such as mTOR. TGF $\beta$ signaling, which is important for the self-renewal of CSCs (Penuelas et al., 2009), can also modulate the expression level of FOXO3A through PI3K/AKT or SMAD signaling (Watabe and Miyazono, 2009). A recent finding that TGF $\beta$ and AKT signaling can cooperatively regulate $\mathrm{FOXO} 3 \mathrm{~A}$, which plays a role in the maintenance of leukemia stem cells, raises the possibility that FOXO3A may be an important mediator in the regulation of GBM CSCs targeted by Z-ajoene (Naka et al., 2010). Interestingly, the 
phosphorylated forms of p38 and ERK were elevated upon treatment with $2.5 \mu \mathrm{M}$ Z-ajoene (Fig. 4). This sharp induction of ERK/p38 activity by Z-ajoene was unexpected but might be attributed, at least in part, to AKT suppression by Z-ajoene and the negative crosstalk between the Erk and PI3K/AKT pathways in GBM CSCs (Sunayama et al., 2010). It has been reported that MAPK ERK and p38 also phosphorylate FOXO3A, resulting in its destabilization (Sunayama et al., 2011). Surprisingly, TGF $\beta$, AKT, ERK, and p38, the expression level of which were modulated by Z-ajoene, all target and downregulate FOXO3A. This pathway-level convergence highlights FOXO3A as one of the key molecules regulating neural stem cell maintenance and integrity. The consequence of these redundant or convergent signaling pathways is that the activation of FOXO3A can mediate a number of transcriptional programs, including NOTCH signaling, which is indispensible for stem cell biology (Kim et al., 2014) The absence of the FOXO3A gene results in a loss of neural stem cells and proper differentiation (Renault et al., 2009). These cells may maintain stem cell properties through the transcriptional regulation of protective autophagy, as in hematopoietic stem cells (Warr et al., 2013), Indeed, the use of FOXO transcription factors as therapeutic agents to target CSCs has been attempted (Zhang et al., 2011).

The perturbation of the mRNA and protein levels of these key genes by Z-ajoene treatment was confirmed, and String 9.1 (http://string-db.org/) (von Mering et al., 2003) was applied to establish a functional/physical interaction map (Supplementary Fig. 2). In addition to the above-discussed molecules, this systems approach uncovered CREB-binding protein (CBP) as a potential interacting hub molecule in the network. However, this in silico analysis must be interpreted carefully because CBP interacts with many transcription factors, such as $\beta$ catenin, to enhance transcription, and it is important for WNT-dependent stem cell proliferation. Two molecules, myosin and TP53inducible glycolysis and apoptosis regulator (TIGAR), were also of interest because they were not associated with other identified molecules in the map. The myosin reported here is phosphorylated non-muscle type II myosin, which is known to be important for human pluripotent stem cell maintenance (Walker et al., 2010) and cell-to-cell interactions. TIGAR primarily functions as a regulator of glycolysis. Little is known about the roles of these proteins in stem cells. The expression levels of myosin and TIGAR were increased and decreased, respectively, by Zajoene in the CSC population. Therefore, their roles in CSC maintenance may need to be investigated.

Taken together, our results demonstrate that Z-ajoene specifically targets CSCs of GBM cells, and this activity may result from decreased AKT activity, increased ERK/p38 activity, and decreased TGF $\beta$ activity, all of which are associated with downregulated FOXO3A expression. Further studies to develop Zajoene as an anti-cancer agent to specifically target the CSC population and to validate its suggested mechanism are warranted. In addition, the systemic approaches demonstrated herein should be used to identify novel anti-CSC agents that avoid nonspecific cytotoxic effects and to effectively identify the potential mechanisms of action of the candidate molecules.

Note: Supplementary information is available on the Molecules and Cells website (www.molcells.org).

\section{ACKNOWLEDGMENTS}

This work was supported by the National Research Foundation of Korea (NRF) grant funded by the Korea government [Ministry of Science, ICT and Future Planning (MSIP)] (2011-
0030074, 2011-0023431 and 2012-M3A9B6055466). We are indebted to TM Kim, for helpful discussion.

\section{REFERENCES}

Bielen, A., Perryman, L., Box, G.M., Valenti, M., de Haven Brandon, A., Martins, V., Jury, A., Popov, S., Gowan, S., Jeay, S., et al. (2011). Enhanced efficacy of IGF1R inhibition in pediatric glioblastoma by combinatorial targeting of PDGFRalpha/beta. Mol. Cancer Ther. 10, 1407-1418.

Block, E., Ahmad, S., Jain, M.K., Crecely, R.W., Apitz-Castro, R. and Cruz, M.R. (1984). The chemistry of alkyl thiosulfate esters. 8. (E,Z)-Ajoene: a potent antithrombotic agent from garlic. J. Am. Chem. Soc. 106, 8295-8296.

Calnan, D.R., and Brunet, A. (2008). The FoxO code. Oncogene 27 2276-2288.

Dean, M., Fojo, T., and Bates, S. (2005). Tumour stem cells and drug resistance. Nat. Rev. Cancer 5, 275-284.

Dirsch, V.M., Gerbes, A.L., and Vollmar, A.M. (1998). Ajoene, a compound of garlic, induces apoptosis in human promyeloleukemic cells, accompanied by generation of reactive oxygen species and activation of nuclear factor kappaB. Mol. Pharmacol. $53,402-407$

Eyler, C.E., Wu, Q., Yan, K., MacSwords, J.M., Chandler-Militello, D., Misuraca, K.L., Lathia, J.D., Forrester, M.T., Lee, J., Stamler, J.S., et al. (2011). Glioma stem cell proliferation and tumor growth are promoted by nitric oxide synthase-2. Cell 146, 53-66.

Fukazawa, H., Mizuno, S., and Uehara, Y. (1995). A microplate assay for quantitation of anchorage-independent growth of transformed cells. Anal. Biochem. 228, 83-90.

Gangemi, R., Paleari, L., Orengo, A.M., Cesario, A., Chessa, L., Ferrini, S., and Russo, P. (2009). Cancer stem cells: a new paradigm for understanding tumor growth and progression and drug resistance. Curr. Med. Chem. 16, 1688-1703.

Gerrard, L., Zhao, D., Clark, A.J., and Cui, W. (2005). Stably transfected human embryonic stem cell clones express OCT4specific green fluorescent protein and maintain self-renewal and pluripotency. Stem Cells 23, 124-133.

Ginestier, C., Hur, M.H., Charafe-Jauffret, E., Monville, F., Dutcher, J., Brown, M., Jacquemier, J., Viens, P., Kleer, C.G., Liu, S., et al. (2007). ALDH1 is a marker of normal and malignant human mammary stem cells and a predictor of poor clinical outcome. Cell Stem Cell 1, 555-567.

Hyun, K.H., Yoon, C.H., Kim, R.K., Lim, E.J., An, S., Park, M.J., Hyun, J.W., Suh, Y., Kim, M.J., and Lee, S.J. (2011). Eckol suppresses maintenance of stemness and malignancies in glioma stem-like cells. Toxicol. Appl. Pharmacol. 254, 32-40.

Kaschula, C.H., Hunter, R., Stellenboom, N., Caira, M.R., Winks, S. Ogunleye, T., Richards, P., Cotton, J., Zilbeyaz, K., Wang, Y., et al. (2012). Structure-activity studies on the anti-proliferation activity of ajoene analogues in WHCO1 oesophageal cancer cells. Eur. J. Med. Chem. 50, 236-254.

Kim, J., Choi, H., Cho, E.G., and Lee, T.R. (2014). FoxO3a is an antimelanogenic factor that mediates antioxidant-induced depigmentation. J. Invest. Dermatol. 134, 1378-1388.

Lee da, Y., Li, H., Lim, H.J., Lee, H.J., Jeon, R., and Ryu, J.H. (2012) Anti-inflammatory activity of sulfur-containing compounds from garlic. J. Med. Food 15, 992-999.

Li, M., Ciu, J.R., Ye, Y., Min, J.M., Zhang, L.H., Wang, K., Gares, M., Cros, J., Wright, M., and Leung-Tack, J. (2002). Antitumor activity of Z-ajoene, a natural compound purified from garlic: antimitotic and microtubule-interaction properties. Carcinogenesis 23, 573-579.

Lu, D., Choi, M.Y., Yu, J., Castro, J.E., Kipps, T.J., and Carson, D.A (2011). Salinomycin inhibits Wnt signaling and selectively induces apoptosis in chronic lymphocytic leukemia cells. Proc. Natl. Acad. Sci. USA 108, 13253-13257.

Malhotra, G.K., Zhao, X., Band, H., and Band, V. (2011). Shared signaling pathways in normal and breast cancer stem cells. J. Carcinog. 10, 38

Mani, S.A., Guo, W., Liao, M.J., Eaton, E.N., Ayyanan, A., Zhou, A.Y., Brooks, M., Reinhard, F., Zhang, C.C. Shipitsin, M., et al. (2008). The epithelial-mesenchymal transition generates cells with properties of stem cells. Cell 133, 704-715.

Matsuda, K., Sato, A., Okada, M., Shibuya, K., Seino, S., Suzuki, K. 
Watanabe, E., Narita, Y., Shibui, S., Kayama, T., et al. (2012). Targeting JNK for therapeutic depletion of stem-like glioblastoma cells. Sci. Rep. 2, 516.

Naka, K., Hoshii, T., Muraguchi, T., Tadokoro, Y., Ooshio, T., Kondo, Y., Nakao, S., Motoyama, N., and Hirao, A. (2010). TGFbeta-FOXO signalling maintains leukaemia-initiating cells in chronic myeloid leukaemia. Nature 463, 676-680.

Penuelas, S., Anido, J., Prieto-Sanchez, R.M., Folch, G., Barba, I., Cuartas, I., Garcia-Dorado, D., Poca, M.A., Sahuquillo, J., Baselga, J., et al. (2009). TGF-beta increases glioma-initiating cell self-renewal through the induction of LIF in human glioblastoma. Cancer Cell 15, 315-327.

Renault, V.M., Rafalski, V.A., Morgan, A.A., Salih, D.A., Brett, J.O., Webb, A.E., Villeda, S.A., Thekkat, P.U., Guillerey, C., Denko, N.C., et al. (2009). FoxO3 regulates neural stem cell homeostasis. Cell Stem Cell 5, 527-539.

Singh, S.K., Hawkins, C., Clarke, I.D., Squire, J.A., Bayani, J., Hide, T., Henkelman, R.M., Cusimano, M.D., and Dirks, P.B. (2004). Identification of human brain tumour initiating cells. Nature 432, 396-401.

Soeda, A., Park, M., Lee, D., Mintz, A., Androutsellis-Theotokis, A., McKay, R.D., Engh, J., Iwama, T., Kunisada, T., Kassam, A.B., et al. (2009). Hypoxia promotes expansion of the CD133-positive glioma stem cells through activation of HIF-1alpha. Oncogene 28, 3949-3959.

Sunayama, J., Matsuda, K., Sato, A., Tachibana, K., Suzuki, K., Narita, Y., Shibui, S., Sakurada, K., Kayama, T., Tomiyama, A., et al. (2010). Crosstalk between the PI3K/mTOR and MEK/ERK pathways involved in the maintenance of self-renewal and tumorigenicity of glioblastoma stem-like cells. Stem Cells 28, 1930-1939.

Sunayama, J., Sato, A., Matsuda, K., Tachibana, K., Watanabe, E., Seino, S., Suzuki, K., Narita, Y., Shibui, S., Sakurada, K., et al.
(2011). FoxO3a functions as a key integrator of cellular signals that control glioblastoma stem-like cell differentiation and tumorigenicity. Stem Cells 29, 1327-1337.

Taylor, P., Noriega, R., Farah, C., Abad, M.J., Arsenak, M., and Apitz, R. (2006). Ajoene inhibits both primary tumor growth and metastasis of B16/BL6 melanoma cells in C57BL/6 mice. Cancer Lett. 239, 298-304.

von Mering, C., Huynen, M., Jaeggi, D., Schmidt, S., Bork, P., and Snel, B. (2003). STRING: a database of predicted functional associations between proteins. Nucleic Acids Res. 31, 258-261.

Walker, A., Su, H., Conti, M.A., Harb, N., Adelstein, R.S., and Sato, N. (2010). Non-muscle myosin II regulates survival threshold of pluripotent stem cells. Nat. Commun. 1, 71.

Warr, M.R., Binnewies, M., Flach, J., Reynaud, D., Garg, T., Malhotra, R., Debnath, J., and Passegue, E. (2013). FOXO3A directs a protective autophagy program in haematopoietic stem cells. Nature 494, 323-327.

Watabe, T., and Miyazono, K. (2009). Roles of TGF-beta family signaling in stem cell renewal and differentiation. Cell Res. 19, 103-115.

Yoon, C.H., Kim, M.J., Kim, R.K., Lim, E.J., Choi, K.S., An, S. Hwang, S.G., Kang, S.G., Suh, Y., Park, M.J., et al. (2012) cJun $\mathrm{N}$-terminal kinase has a pivotal role in the maintenance of self-renewal and tumorigenicity in glioma stem-like cells. Oncogene 31, 4655-4666.

Zhang, S., Balch, C., Chan, M.W., Lai, H.C., Matei, D., Schilder, J.M., Yan, P.S., Huang, T.H., and Nephew, K.P. (2008). Identification and characterization of ovarian cancer-initiating cells from primary human tumors. Cancer Res. 68, 4311-4320.

Zhang, X., Rielland, M., Yalcin, S., and Ghaffari, S. (2011). Regulation and function of FoxO transcription factors in normal and cancer stem cells: what have we learned? Curr. Drug Targets $12,1267-1283$ 